\section{Interview CIRSRad}

Mit dem vollständigen Inkrafttreten des Strahlenschutzgesetzes (StrlSchG) und der Strahlenschutzverordnung (StrlSchV) zum 01.01.2019 müssen Strahlenschutzverantwortliche dafür sorgen, dass Vorkommnisse im Rahmen geplanter medizinischer Expositionsszenarien in ihrer Einrichtung systematisch erfasst und aufgearbeitet werden. Als Vorkommnisse werden Ereignisse bezeichnet, die zu einer unbeabsichtigten Exposition geführt haben, geführt haben könnten oder führen könnten. Erfüllt ein Ereignis darüber hinaus Kriterien für ein „bedeutsames Vorkommnis“, so muss es an die zuständige Behörde gemeldet werden.

Das Bundesamt für Strahlenschutz (BfS) hat zwei Vorhaben initiiert, in denen unter der Leitung des Klinikums Braunschweig und des Klinikums Nürnberg unter anderem eine Software-Lösung zur Erfassung, Aufarbeitung und Meldung von strahlenschutzrelevanten Vorkommnissen in Röntgendiagnostik und Nukle- armedizin entwickelt wurde. Die Software, die unter dem Namen CIRSRad bekannt ist, wird nun, nachdem beide Vorhaben abgeschlossen sind, über die Website der DGMP und der APT einer breiteren Öffentlichkeit zur Verfügung gestellt.

Dr. Markus Borowski (Braunschweig) und Dr. Josefin Hartmann (Nürnberg) geben Auskunft, warum dieses Tool entwickelt wurde und wie es den Alltag in der Röntgendiagnostik und der Nuklearmedizin erleichtern kann.

\section{Wieso ist ein Programm für die Erfassung von strahlenschutzrelevanten Vorkomm- nissen überhaupt notwendig?}

Markus Borowski und Josefin Hartmann:

In der täglichen Routine besteht kaum eine Möglichkeit, ein Vorkommnis, wenn es denn beobachtet wird, unmittelbar bis zum Ende zu bearbeiten. Insbesondere in größeren Einrichtungen wechselt darüber hinaus oft- mals mehrfach am Tag die Besetzung eines Arbeitsplatzes. Da gehen Informationen über Vorkommnisse schnell verloren. Mithilfe der Software ist es möglich, innerhalb weniger Sekunden eine erste Meldung über ein Vorkommnis zu erfassen. Diese kann später vervollständigt und analysiert werden.

Wenn wir von Vorkommnissen sprechen, so meinen wir damit explizit nicht ausschließlich die Ereignisse, die als bedeutsame Vorkommnisse an die zuständige Behörde zu melden sind. Die meisten Vorkommnisse sind nicht meldepflichtig. Sie sind aber entsprechend der StrlSchV innerhalb einer Einrichtung zu erfassen und zu bearbeiten. Innerhalb der Vorhaben konnten wir in den klinischen Einrichtungen, die bei der Anwendungsstudie mitgewirkt haben, erkennen, dass gerade die Vorkommnisse unterhalb der Schwelle zu bedeutsamen Vorkommnissen viel Potenzial haben, um interne Prozesse zu überdenken und zu verbessern. 


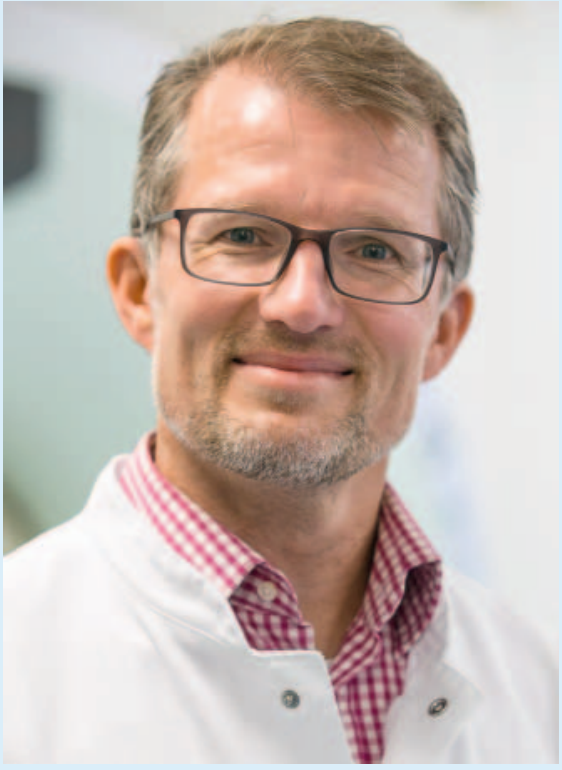

DR. Markus Borowski. (c) Klinikum Braunschweig, P. Sierigk

Welche Vorteile bietet CIRSRad im Vergleich zu anderen Lösungen und wie vereinfacht es die Arbeit von Strahlenschutzbeauftragten?

Zukünftig wird die Erfassung, Bearbeitung und Analyse durch zentrale Softwareprodukte, wie zum Beispiel Dosismanagementsysteme oder RIS-Systeme unterstützt werden. Aktuell sind solche Lösungen meist nicht verfügbar und auch später wird es kleinere Einrichtungen geben, in denen es lange dauert, bis über Softwareupdates die Möglichkeiten zur Erfassung und sinnvollen Bearbeitung von Vorkommnissen verfügbar sind. Andererseits fordern Ärztliche Stellen schon jetzt von allen Einrichtungen ein Konzept zum Umgang mit Vorkommnissen. Die CIRSRad-Software bietet auf elegante Weise eine solche Lösung.

Über die Erfassung von strahlenschutzrechtlich relevanten Vorkommnissen hinaus ermöglicht die CIRSRad-Software im Übrigen auch die Erfassung von weiteren Vorkommnissen, zum Beispiel zu IT-technischen Zwischenfällen oder Geräteproblemen. Hierdurch ermöglicht die Software den lokalen Aufbau einer Wissensdatenbank, in der Anwender bei ähnlichen Problemen später nach Lösungen schauen können.

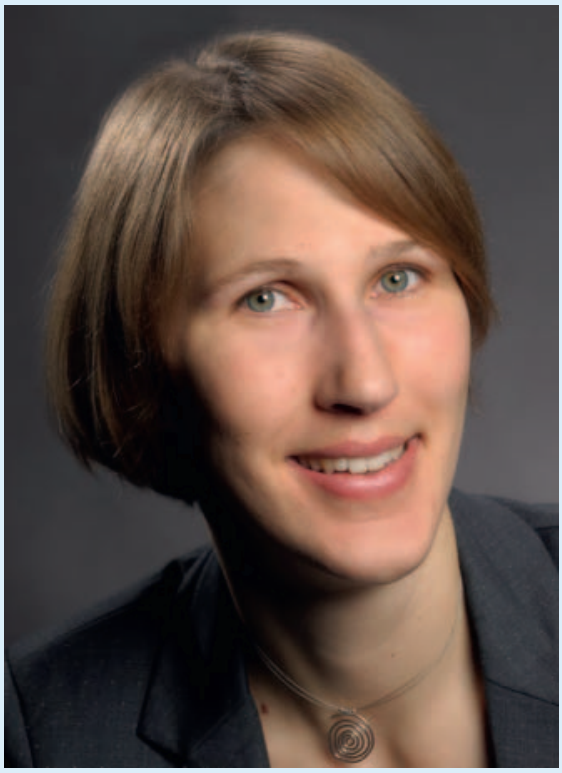

Dr. Josefin Hartmann. (c) Glasow

Für welche Anwendungen in der Röntgendiagnostik und in der Nuklearmedizin ist CIRSRad geeignet?

Wir haben uns bemüht, sämtliche Untersuchungen und Tätigkeiten abzubilden, die im medizinischen Alltag vorkommen. Nachdem in kleineren Einrichtungen aber üblicherweise nur ein Teil aller möglichen Untersuchungsverfahren oder auch nicht alle Gerätetypen verwendet werden, ist es in der Konfiguration der Software möglich, Untersuchungen und Tätigkeiten auszublenden, die vor Ort nicht benötigt werden. Damit bleiben die Dokumentationsmenüs schlank und übersichtlich.

Was muss vor Ort an IT-Infrastruktur vorhanden sein, um mit CIRSRad arbeiten zu können?

Die Software stellt minimale Anforderungen. Einzige notwendige Anforderung an die IT-Infrastruktur ist die Nutzung eines Windows-Betriebssystems. Das sollte aber für die meisten Einrichtungen zutreffen. Dann kann die Software, wie sie auf der Internetseite von DGMP und APT verfügbar ist, einfach in einen Ordner kopiert und ohne Installation genutzt werden. Für größere Einrichtungen empfiehlt es sich, hierzu ein Netzlaufwerk zu verwenden. Das CIRSRad ist auch bedingt Multi-User-fähig. Hierdurch ist es möglich, von unterschiedli- chen Arbeitsplätzen aus parallel an Vorkommnissen zu arbeiten und Einträge vorzunehmen.

Gibt es Schnittstellen zu bereits bestehenden Systemen oder müssen die Daten manuell eingeben werden?

Wir wollten das System so einfach und schlank wie möglich halten. Auch hoffen wir, dass Vorkommnisse, auch unterhalb der Schwelle zu bedeutsamen, meldepflichtigen Vorkommnissen, nicht häufig auftreten. Die Anwendungsstudie, die wir durchgeführt haben, hat das auch so gezeigt. Daher wurden keine aufwändigen Schnittstellen zu anderen Systemen etabliert. Die wenigen Parameter, die zur Erfassung und Bearbeitung eines Vorkommnisses erforderlich sind, müssen somit schon eingegeben werden.

Wie vereinfacht CIRSRad die Kommunikation mit den zuständigen Behörden?

Insbesondere bedeutsame Vorkommnisse werden in den meisten Einrichtungen nur sehr selten auftreten. Sollte ein derartiges Vorkommnis auftreten, stellt sich vielfach die Frage, was alles in welcher Form der zuständigen Behörde zu übermitteln ist. Das CIRSRad ermöglicht es, anhand der im Rahmen der Bearbeitung eines Vorkommnisses erfassten Daten, ein Schreiben für die jeweilige Behörde zu erstellen. In der Konfiguration der Software kann hierzu auch zum Beispiel die Anschrift der Behörde hinterlegt werden.

An welche Nutzer richtet sich die Software? Ist sie für Medizinphysikexperten, Ärzte, MTRA oder Strahlenschutzbeauftragte der Einrichtungen gedacht?

Die Erfassung, Bearbeitung und Analyse von Vorkommnissen schließt alle gerade genannten Personengruppen und potenziell noch weitere mit ein. Das CIRSRad unterstützt alle diese Berufsgruppen in den für sie spezifischen Aufgabenteilen, die in jeder Einrichtung je nach Größe und Struktur, variieren können. Beispielsweise unterstützt die Software MTRA bei der ersten Erfassung eines Vorkommnisses und der Beschreibung des Ereignisses, Medizinphysikexperten bei der Bewertung der Höhe der Exposition des jeweiligen Patienten so- 
wie gegebenenfalls Überlegungen zu Optimierungen, um derartige Vorkommnisse in der Zukunft zu vermeiden und Strahlenschutzbeauftragte bei der Analyse, der Festlegung von Gegenmaßnahmen sowie auch potenziell der Erstellung von Schreiben an die zuständige Behörde.
Unabhängig von der Software wurde innerhalb der Vorhaben im Übrigen auch ein Leitfaden erarbeitet, der exemplarisch für unterschiedliche Einrichtungstypen sinnvolle Vorgehensweisen zum Umgang mit Vorkommnissen enthält. Zudem werden darin typische Aufgaben der einzelnen Per- sonengruppen vorgestellt. Dieser Leitfaden ist ebenso über die Internetseite von DGMP und APT verfügbar.

Sie möchten mehr erfahren? Dann schauen Sie vorbei auf: www.apt.drg.de > Fachinformationen > CIRSRad 